\title{
Use of coaxial plasma guns to start up field-reversed-mirror reactors
}

\author{
A. C. Smith, Jr. \\ G. A. Carlson \\ J. L. Eddleman \\ C. W. Hartman \\ W. S. Neef, Jr.
}

March 19, 1980 


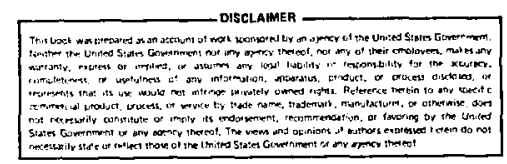

Use of coaxial plasma guns to start up field-reversed-mirror

\section{reactors}

A. C. Smith, Jr.*

G. A. Carlson

J. L. Eddleman

C. W. Hartman

W. S. Neef, $\mathbf{J}$ r.

Manuscript date: March 19, 1980

* Pacific Gas and Electric Company, San Francisco, Californja.

LAWRENCE LIVERMORE LABORATORY University of California * Livermore, California • 94550 


\title{
Use of coaxial plasma guns to start up field-reversed-mirror \\ reactors
}

\begin{abstract}
Application of a magnetized coaxial plasma gun for start-up of a fieldreversed-mirror reactor is considered. The design is based on preliminary scaling 1 aws and is compared to the design of the start-up gun used in the Beta II experiment.
\end{abstract}

\section{NTRODUCTION}

Several recent conceptual designs of fusion reactors have been based on field-reversed mirror plasmas $(1,2,3)$. In the designs of Refs. 1 and 2 the field-reversed plasma density, energy, and current a re sustained in steady state by neutral-beam injection, while in Ref. 3, field-reversed plasma rings are $f e d$ in at one end of the reactor, buin as they move down the reactor core, and are exhausced at the discharge end of the reactor.

A neceseary precursor to the plasma burn, be it sustained in steady state or transient, is the creation of the field-reversed-mirror plasma. In Ref. 1 a number of possible start-up scenarios were qualitatively degcribed: "slow buildup" with neutral beams, "fast" ion injection with an intense, pulsed, ion-neutral source (IP INS), a reversed-field theta-pinch, rotating el ectron or ion beams, conical theta-pinch guns, and a magnetized coaxial plasma gun.

Subsequent to the publication of Ref. 1, the magnetized coaxial plasma gun was chosen as the preferred field-reversal start-up method for the Beta II experiment at Iawrence Li vermore Laboratory. Initial experimental results on the Beta II device $(4,5)$ have demonstrated production of field-reversed flux and have extended the pioneering work of Alfven and co-workers (6) to a fieldreversed flux useful for starting up present-day field-reversed plasma experiments. Experiments on Beta II are proceeding to the next stage of axial translation and wirror trapping of field-reversed plasma, to be followed by 
neutral-bea injection. In this report we consider the design (based on preliminary scaling laws) of a coaxial plasua gun for start-up in the fieldreversed-mirror pilot reactor of Ref. 2 .

THE MAGNETIZED COAXIAL PLASMA GJN

The basic arrangement for formation of field-reversed plasma rings with a coaxial plasma gun is shown schematically in Fig. la. The plasma gum is a cylindrically-sydretric, annular-breeched device in which a ring-shaped plasma is formed and accelerated by discharge current flowing radially across the anode-cathode gap. If the gun's external solenoid windings and the solenoid windings inside the center electrode are properly designed, the central, axial magnecic flux can be constrained largely within the center el ectrode. The field lines emerge radially at the ends of the center electrode and retum outside the body of the gun, forming a magnetic-cusped field at the muzzle end of the $\mathrm{gm}$.

While the axial magnetic field in the anode-cathode gap may be quite small, a tomidal field will be imbedded in the plasma during acceleration owing to the large, axial current flowing in the center electrode. As the plasma lesves the $\mathrm{g}$ in it encounters the radial-field component of the magnetic cus $\mathrm{p}$, as shown in Fig. 1b. Inertia and $J \times \mathrm{B}$ acceleration, together with high conductivity, cause the plasma to "push" the radial field, thus capturing magnetic flux as it passes through the cusp. Behind the ring the extended field lines break and reconnect so that the ring emerges downtream from the cuep-with its "captured $f l u x "$ - $2 s$ an amular plasma confined by an axially field-reversed, closed magnetic field. The plasma ring may subsequent $1 y$ drift into a magnetic-mirror trough where it could be trapped and a fusion plasma bum initiated, as shown in Fig. lc. Moreover, upon leaving the gun, the plasma could also be made to enter a region of increasing axial field for the purpose of heating the fusion fuel by magnetic compression prior to confinement in an axial-airror well.

\section{GUN START-UF IN THE BETA II EXPERIMENT}

The construction of a magnetized coaxial plasma gun for starting up a field-reversed plasma in Beta II was proposed in August 1978 (7). The goals of the experimental program on Beta II were atated to be: (a) formation of the 


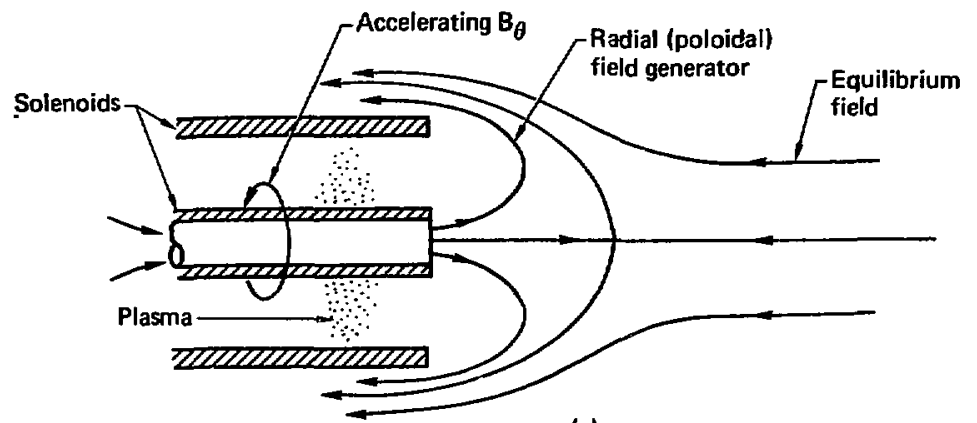

(a)

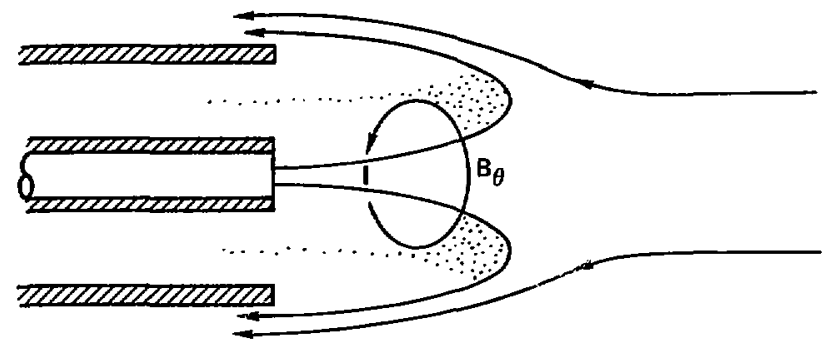

(b)

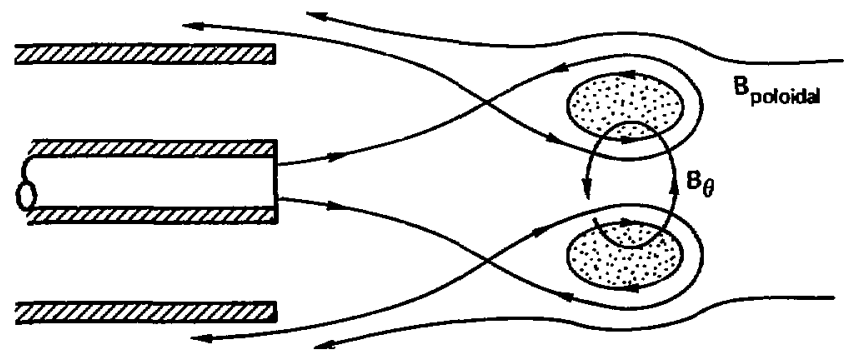

(c)

Fig. 1. Foration of field-reversed plasma by magnetized coaxial pl asma gun during (a) acceleration; (b) elongation; and (c) reconnection. 
field-reversed plasma, (b) translation and magnetic mirror trapping of the plasma, and (c) neutral-beam sustenance of the plasma.

Achievement of the first goal was reported in November 1979 (4). Internal, magnetic-probe measurements have demonstrated production of fieldreversed plasma for a variety of experimental conditions. For one set of data the equilibrilm guide $i$ ield was $B_{o}=4.8 \mathrm{kG}$ and a probe signal $\frac{\Delta B}{B_{o}}=3.1$ was recorded corresponding to a reversed field $B_{z}=-10 \mathrm{kG}$. The radius of fieldreversed plasma was in the range 10 to $15 \mathrm{~cm}$.

Continuing experiments on Beta II will attempt to trap the field-reversed plasma in a ragnetic mirror. Here, the emphas is will be on quasi-static methods which will be applicable to the superconducting magnet envi ronment of a fusion reactor.

\section{GUN START-UP FOR THE FIELD-REVERSED-MIRROR P DOT REACTOR}

The field-reversed-mirror pilot reactor (2) is shown in Fig. 2. The field-reversed DT plasma is at the center of the reactor. A cylindrical blanket and shield surround the plasma. Sur rounding the blanket and shield is a set of niobium-titanium superconducting magnets which provide the required background magnetic field. Neutral-beam injectors fuel and sustain the energy of the plasma. The large tanks at the ends of the reactor house direct energy converters and vacuum-pumping systems for the plasma leakage. 'ihe coaxial plasma gun for stgrt-up is located at one end of the reactor, inside the di rect-converter tank.

During start-up the poloidal-field generator coils of the gun would be pulsed. The gun would be positioned so that the fringe field of the reactor acts as an increasing guide field to remove, by self-compresaion, near $1 \mathrm{y}$ all the kinetic energy of the ring. After self-conpression the ring would drift (at low kizetic energy) into the neutral-beam region where the equilibrium position would be established by weak time-varying fields, by a nearby dissipative structure to damp the ring motion, or possibly by dissipation within the ring itself.

During the plasma burn (gun coils off), charged particles diffusing out of the field-reversed plasma region will stream al ong open magnetic-field lines to the direct converters. To permit uninhibited passage of the escaping plasma strean to the direct converters, the gun uses a hollow center el ectrode with an inside radius that exceds the radius of the magnetic-field-line bundle carrying plasma. 


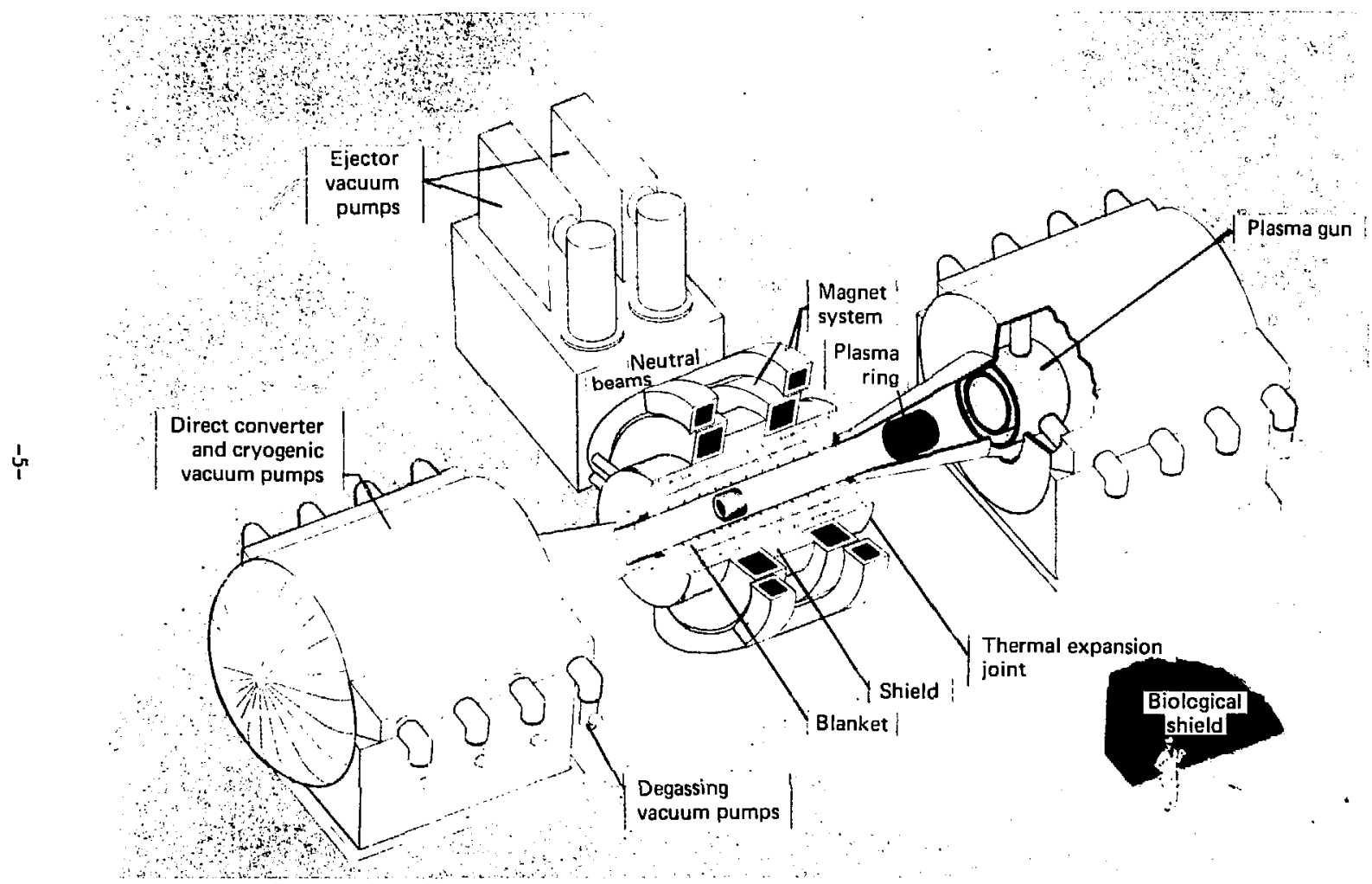

Fig. 2. Field-reversed-mirror pilot reactor. 
The predicted performance of a field-reversed-mirror plasma depends strongly on the parameter $S$, the ratio of plasma mirror radius, $a$, to the ion gyroradius, $\rho_{i}$. Stability of the plasma is believed to require that $s$ be $a$ small number, probably 10 or less. For the field-reversed-mirror pilot reactor, both $s=5$ and $s=7$ were considered, and the reactor was designed to accommodate either plasma. The steady-state-fusion plasma ring is maintained by neutral beams inside the reactor and is assumed to be a spherical Hill's vortex with the characteristics given in Table 1.

Table 1. Plasma parameters for field-reversed-mirror pilot reactor.

\begin{tabular}{|c|c|c|c|}
\hline Par ameters & & $s=5$ & $s=7$ \\
\hline $\mathbf{T}_{\mathbf{i}}, \mathrm{keV}$ & $=$ & 67 & 102.8 \\
\hline$T_{e}, k e v$ & $=$ & 35 & 59.7 \\
\hline $\mathrm{U}_{\text {ions }}, \mathrm{MJ}$ & $=$ & 0.49 & 2.64 \\
\hline $\mathrm{U}_{\text {el ectrons, }}$ & $=$ & 0.26 & 1.53 \\
\hline $\mathbf{U}_{\text {the rmal }}, \mathrm{MJ}$ & $=$ & 0.75 & 4.17 \\
\hline Umagnetic, MJ & $=$ & 0.056 & 0.31 \\
\hline$I_{\text {toroidal }}, \mathrm{MA}$ & $=$ & 4.59 & 8.05 \\
\hline$\psi_{\text {poloidal }}, \mathrm{Hb}$ & $=$ & 0.237 & 0.755 \\
\hline$N_{\text {ions }}$ & $=$ & $3.08 \times 10^{19}$ & $1.08 \times 10^{20}$ \\
\hline Byacuum, $\mathbf{T}$ & $=$ & 4.79 & 4.58 \\
\hline $\mathbf{R}_{\text {fina } 1, m}$ & $=$ & 0.123 & 0.224 \\
\hline
\end{tabular}

In Table 1, umagnetic was evaluated by using the fact that, for a spherical Hi1l's vortex,

$$
8=U_{\text {thermal }} / U_{\text {magnetic }}=27 / 2 .
$$

The toroi dal current,

$$
I_{\text {toroidal }}=\frac{20 \sqrt{2}}{3 \pi} \frac{\Psi_{\text {poloidal }}}{\mu_{D_{\text {fi nal }}}},
$$


and the poloidal flux, $\psi_{\text {poloidal }}$, were evaluated by noting that, for the spherical Hill's vortex,

$$
\mathrm{U}_{\text {thermal }}^{\mathrm{pl} \text { asma }}=\frac{32 \sqrt{2}}{7 \pi} \frac{\psi_{\text {pol oidal }}^{2}}{\mu_{\mathrm{o}} \mathrm{R}_{\mathrm{final}}} .
$$

Because coaxial pl asma guns typically accelerate plasma to $1-10 \mathrm{keV}$ energy, it is unlikely that the injected ring can be made to have the desired steady-state temperatures (for $S=5(7), T_{i}=67$ (103) keV and $T_{e}=35$ (60) keV). Further, if a scal ed-up gun performs like present 1 aboratory-scale guns, a substantial toroidal field, $B_{\theta}$, will be embedded in the ring during production. We have developed an injection scenario which is bas ed on the presence of a toroidal field and which addresses the mismatch of injected and steady-state ring parameters ( 8 ). Assuming that the gun produces a relatively low- temperature and 1 ow $B$ plasma, the approximate equilibrium requi rement (Bennett relation),

$$
I_{\text {toroi dal }}^{2} \simeq \mathrm{ne}\left(\mathrm{T}_{\mathrm{e}}+\mathrm{T}_{\mathrm{i}}\right) \mathrm{r}_{\mathrm{p}}^{2}+\frac{\mathrm{B}_{\theta}^{2}}{2 \mu_{0}} \mathrm{r}_{\mathrm{p}}^{2},
$$

can be satisfied initially by the toroidal-field-pressure term above. After the ring has been positioned in the beam region, the plasma-pressure term above can be increased by neutral-beam heating whil = $B_{\theta}$ is allowed to decay resistively. In this way the ring size and equilibrim requirements remain approximately constant during heating.

Self-compression of the ring as it drifts along the guide field into the neutral-bean region can be estimated by noting that the poloidal flwx $\psi_{p}$ is conserved in the high conductivity 1 imit. We assume that during self-compression, and with embedded toroidal field, the ring remains approximatel y a spherical Hill's vortex with plasma thermal energy and toroidal field energy related by the constant ratio, $g$. With these assumptions, we may write the energy balance before and after compression as

$$
v_{\text {thermal }}^{\mathrm{c}}\left(1+\frac{1}{g}\right)+v_{\text {kinet ic }}^{o}=v_{\text {thermal }}^{f}\left(1+\frac{1}{g}\right)+v_{\text {kinetic }}^{f} \text {, }
$$

where the superscripts " $o$ " and " $f$ " refer to initial and final conditions, respectively. If the magnetic flux inside the ring is conserved during compression, then the condition under which a ring comes to rest as it moves into a stronger magnetic field, $B^{f}$, is given by 


$$
u_{\text {kinetic }}^{o}=u_{\text {thermal }}^{f}\left(1+\frac{1}{g}\right)\left[1-\left(B^{0} / B^{f}\right)^{1 / 2}\right] .
$$

This condition for stopping the ring leads to the expression for the fraction of total ring-plasma energy that is at first axially-directed kinetic energy:

$$
f=\frac{v_{k i n e t i c}^{o}}{v_{\text {kinetic }}^{o}+v_{\text {magnetic }}^{o}+v_{\text {thermal }}^{o}}=\left[1-\left(B^{\circ} / B^{f}\right)^{1 / 2}\right] \text {. }
$$

The axial magnetic-field strength is plotted in Fig. 3 as a function of 2 , the distance into the direct converter for both the $S=5$ and the $S=7$ cases. The radial distance from the machine's axis to the flux lines that lie just outside the separatrix, $R_{f l u x}$, is plotted in Fig. 4, also as a function of $z$. We have used the analytic assumptions above and the data shown in Figs. 3 and 4 , to plot the initial radius, $R^{\circ}$, of a plasme ring formed at a distance 2 into the direct-converter region that comes to rest at the reactor mirrm we11; Figs. 5 and 6 show the plots for the $S=5$ and $S=7$ cases, respectively. Also shown in Figs. 5 and 6 is the behavior of the function " $f . "$

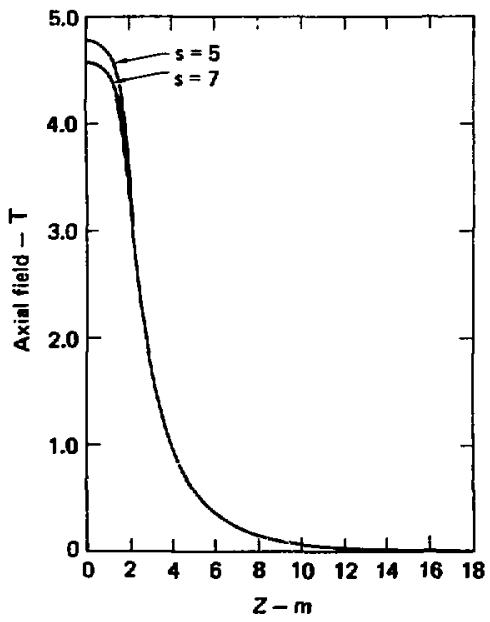

Fig. 3. Axial magnetic field strength on reactor axis.

To pernit uninhibited passage of the escaping plasma stream to the direct converter, our gun design incorporates a hollow center electrode having a radius wich exceeds $R_{\text {flux }}$ for the $S=7$ case. Since the gun may 


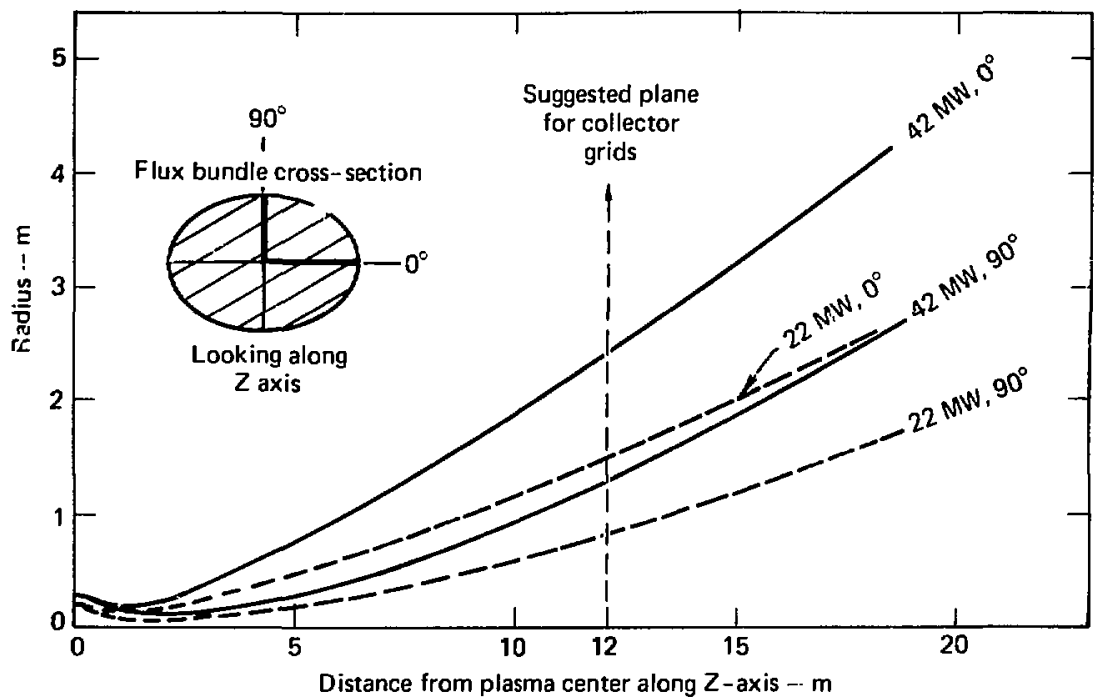

Fig. 4. Magnetic field lines at plasma limit.

well have a length of 1-2 $\mathrm{m}$, the bore of the hole at the rear of the center electrode must be large enough to miss the exiting plasma stream. The refore, if the hole in the center electrode is right-cylindrical, the plasma rings formed in the gun will be larger than equilibirium radius for either plasma case (see Figs. 5 and 6). The radial pressure imbal ance is sustained by the center electrode and as the rings leave the muzzle of the gun they shrink in radius to the equilibrium val w, $\mathbf{R}_{0}$. Experimental determination of the val ue of " $f$ " achievable in these guns would then dictate the axial location and radial size of the $g \mathrm{~m}$. For example, if $f \simeq 0.5$, the muzzle of the gun would have to be located at roughly $z=3.8 \mathrm{~m}$, with the hole in the center el ectrode having a radius greater than $50 \mathrm{~cm}$ to avoid intersecting the $\mathrm{S}=7$ plasma stream at its widest point.

A current model of the gun operation (8) has the acceleration of plasma taking place largely after the current-sheath-rundown phase. At the end of rundown of the current sheath it is believed that $99 \%$ of the gas has been pushed radially outward againgt the outer el ectrode while the remaining 17 of the gas is trapped as a low- $\beta$ plasma in the high- $B_{\theta}$ field region near the 

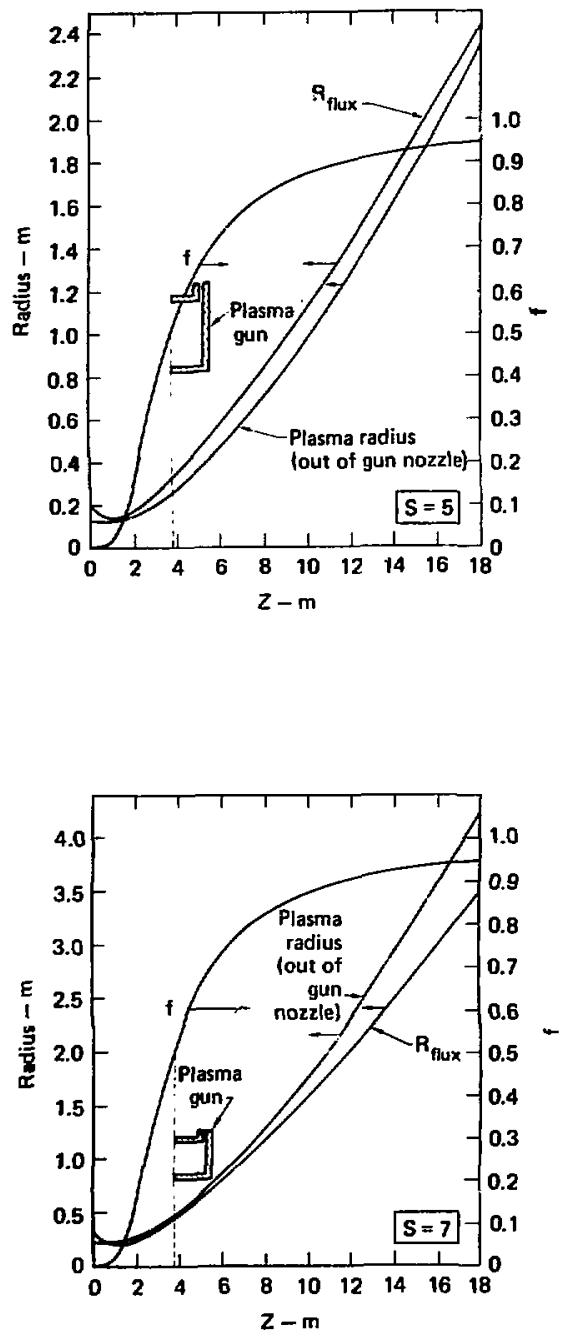

Fig. 5. Initial plasma radius and energy function $f, S=5$ plasma.
Fig. 6. Initial pl asma radius and energy function $f, s=7$ pl asma. 
center electrode. The subsequent acceleration into a vacuu takes place as a magnetosonic rarefaction wave propagates towards the gun breech. The expansion converts the internal energy, $\sim \mathrm{B}_{0}^{2} / 2 \mu_{0}$, into streaming-pl asma kinetic energy. The low-mass, low- $\beta$ plasma is promptly ejected as a high-energy component (HEC). This is followed by ejection of a low-energy plasma component over a long period of time. The two plasma components each have about one-half the input energy of the gun, and for an operating 200-kJ gun (9) the HEC forms a "slug" of plasma with a length about equal to that of the gun.

In the gun application considered here, a poloidal fringe field inhibits the free expansion of plasma beyond the muzzle. If the poloidal-field pressure is small compared to the stagnation pressure of the HEC, nearly free expansion occurs, and if field reconnection takes place behind the HEC "sl ug" roughly $50 \%$ of the gun energy would be trapped in the ring (mostly as kinetic energy). This energy could be converted into plasma pressure and magnetic energy by self-compression as the ring drifts along an increasing guide field. For this case the overall snergy efficiency of ring production might be $\sim 50 \%$.

Numerical cal culations in progress (10) suggest that if the poloidalfield pressure approaches the HEC stagnation pressure, the kinetic energy of the HEC can be reduced and probably made smali. For this case, if the HEC expands to a length $\approx 1$, reducing $B_{\theta}$ by 2 (existing gurs tend to selfcrowbar after rundown and thus conserve toroi dal flux) before reconnertion occurs, three-fourths of the HEC energy would be trapped in the ring to give an overall efficiency of $37.5 \%$. For this case the energy would be most $1 \mathrm{y}$ magnetic since the HEC is $10 \mathrm{w}-\beta$.

The foregoing discussion suggests that the overall energy efficiency of ring production can be in the range $30-50 \%$. In this report the efficiency wiII be assumed to be $25 \%$.

Marshal has considered scaling liws for coaxial guns (8). Basing his argument on the mechanisms discussed ear lier (f or which the output energy of the gun is equal to the magnetic energy stored in the gun at completion of rundown), he finds

$$
\mathrm{u}=\frac{1}{2} \mathrm{~L}_{\mathrm{g}} \mathrm{I}^{2}=12.53 \mathrm{l}_{\mathrm{g}}(\mathrm{cm}) \mathrm{v}(\text { volts }) \mathrm{r}_{\mathrm{l}}(\mathrm{cm}) \sqrt{\rho\left(\mathrm{gm} \mathrm{cm}^{-3}\right)} \mathrm{J},
$$

where $I_{g}$ is the gun length, $V$ the terminal voltage, $r_{1}$ the center electrode radius, and $\rho$ the filling-gas density. 
Forming an $\mathbf{S}=5 \mathrm{pl}$ asma ring having $\mathrm{U}_{\mathrm{T} \text { otal }}=6.75 \mathrm{MJ}+0.056 \mathrm{MJ}$ and the assumed efficiency $n=25 \%$ would require a gun energy of U gun $-3.2 \mathrm{MJ}$. Taking $p=1.17 \times 10^{-7} \mathrm{~g} / \mathrm{cm}^{3}\left(1-T\right.$ orr $\left.D_{2}\right)$, the above scaling 1 aw gives $1_{\mathrm{g}} \mathrm{Vr}_{1}=7.47 \times 10^{8} \mathrm{~V} \cdot \mathrm{cm}^{2}$. For the $\mathrm{s}=7$ case (U total $=4.48 \mathrm{MJ}$ ), the scaling 1 aw gi ves $1 \mathrm{~g}^{\mathrm{Vr}} \mathrm{r}_{1}=4.18 \times 10^{9} \mathrm{~V} \cdot \mathrm{cm}^{2}$. An arbitrary choice of $f=0.5$ lacates the $\mathrm{gm}$ (as discussed above) at $z \sim 3.8 \mathrm{~m}$. To accommodate an adequate hole in the center electrode (see Figs. 5 and 6), we choose the center el ectrode $\mathrm{radi}$ us to be $r_{1}=85 \mathrm{~cm}$. (The choice of the inner radius of the outer electrode is somewhat arbitrary, but $\mathbf{r}_{2} \simeq \mathbf{r}_{1}+30 \mathrm{~cm} \simeq 115 \mathrm{~cm}$ would probably be reasonable.) Because $r_{1}$ exceeds the ring's equilibrium radius in the megnetic field just beyond the end of the $\mathrm{gm}$, the ring compresses immediatel $y$ after leaving the gun muzzle, as we have expi ained above. A gun length $l_{g}=150 \mathrm{~cm}$ would permit the $s=7 \mathrm{pl}$ asma effluent al so to clear the rear end of a right-cylindrical hole in the center el ectrode. A gun having these dimensions is shown schematically in Figs. 5 and 6 . From the expression above, we obtain a gun terminal voltage $V=59 \mathrm{keV}$ to initiate the $S=5$ plasma, while the $S=7 \mathrm{pl}$ asma would require $\mathrm{V} \simeq 330 \mathrm{keV}$. A comparison of these predicted reactor-gun characteristics with the characteristics of the gun desi gned for the Beta II experiment appears in Table 2.

Table 2. Comparison of Beta II gun and reactor $\mathrm{gm}$.

\begin{tabular}{|c|c|c|c|}
\hline & & Isma gun & \\
\hline & Beta II gun & $S=5$ & $s=7$ \\
\hline Center electrode radius, $\mathrm{R}_{1}$, cm & 7.5 & 85 & 85 \\
\hline Outer electrode radius, $\mathrm{R}_{2}$, $\mathrm{cm}$ & 15 & 115 & 115 \\
\hline Length, ${ }^{1} g, \mathrm{~cm}$ & 150 & 150 & 150 \\
\hline Vol tage, $\mathrm{v}, \mathrm{kv}$ & 40 & 59 & 330 \\
\hline Out put energy, $\mathrm{U}_{g}, \mathrm{MJ}$ & 0.2 & 3.2 & 17.9 \\
\hline Flux in center electrode, $\psi_{\text {poloidal, }}$ wb & 0.07 & 0.24 & 0.755 \\
\hline Poloidal B in center electrode, $B_{\mathrm{p}}, T$ & 4.0 & 0.104 & 0.33 \\
\hline Guide field at gun muzzle, $T$ & $0.2-0.4$ & 1.1 & 1.05 \\
\hline
\end{tabular}


From the preliminar y cal cul ations given above, it appear that a coaxial plasma gur wight scale ressonably to reactor-grade operating conditions. Ongoing experiments and numerical simulations should shed some 1 ight on the validity of the scaling 1 as described above. 


\section{REFERENCES}

1. G. A. Carlson et al., Coneptual Design of the Fiel d-Reversed Mirror Reactor, Lawrence Li vermore Laboratory, Li vermore, CA, UCRL-52467 (1978).

2. G. A. Carlson, K. R. Schultz and A. C. Smith, Jr., Definition and CanCeptual Design of a Small Fusion Reactor, subritted for publication to Electric Power Research Institute.

3. A. C. Smith, Jr., et al., Preliminary Design of the Moving Ring FieldReversed Mirror Reactor, Pacific Gas \&lectric Report 78-Fus 1 (1978).

4. W. C. Turner, C. W. Hartman, J. W. Shearer, J. Taska, "Fiel d Reversed Plasma Gun Experiment", Bul1. Am. Phys. Soc., 24, 1083 (1979).

5. W. C. Turner, C. W. Hartman, J. Taska, A. C. Smith, Jr., Initial Results of Fiei d-Reversed plasma Gu Experiment, Lawrence Livermore I,aboratory, Li vermore, CA, UCRL-83687 (1979).

6. H. Alfvén, L. Lindberg, P. Mitlid, J. Nucl. Energy, 1, 116, (1960).

7. T. C. Simonen, Plasme Gun Proposal, Lawrence Li vermore Laboratory, Livermore, CA, LLL-P ro p-156 (1978).

8. J. Marshall, "Coaxial Guns as Injectors of High Linear Theta Pirches," Proc. of the High Beta Works hop at LASL, Jul y 28-A ugust 1, 1975, ERAS-76/108(1976), P. 470 .

9. I. Henmins and J. Hamel, Bull. Am. Phys. Soc. 22, 1115 (1977).

10. J. Shearer et al., Paper 3E9, 1979 IEEE International Conference on Plasme Science, June 4-6, 1979, IEEE Catal og No.: $79 \mathrm{cH} 1410$-ONPS. 